Research Article

\title{
Comparative Analysis of Free Amino Acids and Nucleosides in Different Varieties of Mume Fructus Based on Simultaneous Determination and Multivariate Statistical Analyses
}

\author{
Jinmei Ou $\mathbb{D}^{1,2}$ Rui Wang, ${ }^{1}$ Xiaoli Li, ${ }^{1}$ Luqi Huang $\mathbb{D},{ }^{2}$ Qingjun Yuan, ${ }^{2}$ Chengwu Fang, \\ and Deling $\mathrm{Wu}^{1}$ \\ ${ }^{1}$ Anhui University of Chinese Medicine, Heifei 230038, China \\ ${ }^{2}$ China Academy of Chinese Medical Sciences, Beijing 100700, China \\ Correspondence should be addressed to Luqi Huang; huangluqi01@126.com
}

Received 30 January 2020; Revised 15 June 2020; Accepted 24 June 2020; Published 1 August 2020

Academic Editor: Kevin Honeychurch

Copyright (c) 2020 Jinmei Ou et al. This is an open access article distributed under the Creative Commons Attribution License, which permits unrestricted use, distribution, and reproduction in any medium, provided the original work is properly cited.

\begin{abstract}
Mume Fructus (MF) contains a variety of organic acids, free amino acids, and nucleoside components, and studies have not yet analyzed the relationship between the components of free amino acids and nucleosides with the varieties of MF. A rapid and sensitive method was established for simultaneous determination of 21 free amino acids and 9 nucleosides in MF by ultrafast liquid chromatography-mass spectrometry. The analysis was carried out on a Waters XBridge Amide column $(100 \mathrm{~mm} \times 2.1 \mathrm{~mm}$, $3.5 \mu \mathrm{m}$ ) with elution by the mobile phase of $0.2 \%$ aqueous formic acid (A) and $0.2 \%$ formic acid acetonitrile (B) at a flow rate of $0.2 \mathrm{~mL} / \mathrm{min}$ with $1 \mu \mathrm{L}$ per injection. The column temperature was maintained at $30^{\circ} \mathrm{C}$. The target compounds were analyzed by the positive ion multiple reaction monitoring (MRM) mode. The comprehensive evaluation of the samples was carried out by principal component analysis (PCA) and technique for order preference by similarity to an ideal solution (TOPSIS) analysis. Results showed the method could simultaneously determine 30 components in MF. The content of total analytes in six mainstream varieties was different, exhibited the order Nangao $>$ Daqingmei $>$ Zhaoshuimei $>$ Yanmei $>$ Shishengme $>$ Baimei, and aspartic acid and adenosine were the most abundant amino acid and nucleoside. PCA and OPLS-DA could easily distinguish the samples, and 11 components could be chemical markers of sample classification. TOPSIS implied that the quality of Nangao and Daqingmei was superior to the other varieties. The results could provide a reliable basis for quality evaluation and utilisation of medicinal and edible MF.
\end{abstract}

\section{Introduction}

Mume Fructus (MF) is derived from the immature fruit of Prunus mume Sieb. et Zucc., named Wumei (in China), and is commonly used food and Chinese medicines, with astringing the lung to stop cough, relieving diarrhea with astringents, removing toxicity for detumescence, promoting fluid relieving thirst, and calming Ascaris [1, 2]. Modern pharmacological studies have shown that it has antibacterial, anti-Alzheimer, antiulcer, antitumor, antivirus, and other biological activities [3-6]. For its important medicinal and edible processing value, $\mathrm{MF}$ is widely cultivated in China $[7,8]$, and the medicinal varieties are mostly distributed in
Southwest areas of Sichuan and Yunnan provinces. In the preliminary investigation, some edible varieties were mixed as medicinal varieties, and the differences in appearance and internal components of varieties brought many problems to the identification and quality evaluation of MF [9].

Phytochemical studies have revealed that MF contains various components, including organic acids, amino acids, nucleotides, terpenoids, inorganic elements, volatile components, and polysaccharides [2, 10]. Organic acids are known as bioactive constituents, and amino acids and nucleotides also play synergistic roles with organic acids in the clinical effects of MF [11, 12]. Amino acids are the basic building blocks of proteins which are required for human 
nutrition $[13,14]$; $L$-methionine, $L$-threonine, $L$-arginine, and glycine have beneficial effects on intestinal flora; the conclusion may explain the obvious antidiarrhea effect of MF [15]. Nucleosides have important physiological functions, such as adenosine can treat angina pectoris, myocardial infarction, cerebrovascular disorders, and other diseases [16]. Scholars have used the components of amino acids and nucleosides to evaluate the quality of medicines and edible medicinal plants [17-19], and the results showed that differences in the composition and contents of amino acids and nucleosides could be used as biomarkers to characterize the quality of medicines and food. Until now, few studies have focused on the components of amino acids and nucleosides in MF; the methodologies of the amino acid analyzer and PITC-HPLC methods mainly focus on quantification $[20,21]$ and have some deficiencies in resolution and separation time.

In the current study, an ultrafast liquid chromatographymass spectrometry (UFLC-MS/MS) method was developed for the determination of 21 amino acids and 9 nucleosides in $\mathrm{MF}$, and then the contents in six mainstream varieties were compared and analyzed by the multivariate statistical analysis method (including principal component analysis (PCA) $[22,23]$ and technique for order preference by similarity to an ideal solution (TOPSIS) method $[24,25]$ ). The proposed method can provide theoretical basis for the quality evaluation and comprehensive utilisation of MF and other edible medicines.

\section{Materials and Methods}

2.1. Chemicals, Reagents, and Materials. Thirty chemical standards were used: $L$-isoleucine (1), $L$-histidine (2), $L$-phenylalanine (3), $L$-leucine (4), $L$-glutamic acid (5), $L$-tyrosine (6), $L$-alanine (7), $L$-cystine (8), $L$-threonine (9), $L$-methionine (10), $L$-proline (11), $L$-aspartic acid (12), $L$-asparagine (13), $L$-serine (14), glycine (15), $L$-valine (16), $L$-arginine (17), $L$-hydroxyproline (18), $L$-tryptophan (19), $L$-lysine hydrochloride (20), $\gamma$-aminobutyric acid (21), uracil (22), adenine (23), cytidine (24), inosine (25), thymidine (26), guanosine (27), adenosine (28), uridine (29), and hypoxanthine (30). The purity of all standard components was $\geq 98 \%$. The structures of these standard substances are shown in Figure S1. Among them, $L^{-}$ isoleucine, $L$-histidine, $L$-phenylalanine, $L$-leucine, $L$-glutamic acid, $L$-tyrosine, $L$-alanine, $L$-cystine, $L$-threonine, $L$-methionine, and $L$-proline were purchased from the National Institutes for Food and Drug Control (Beijing China). The remainder were obtained from Shanghai Yuanye Biotechnology (Shanghai, China). Chromatography-grade methanol and acetonitrile were purchased from Merck (Darmstadt, Germany). Ultrapure water was produced by the Milli-Q purification system (Millipore, MA, USA).

These samples were collected from May to July 2018 in various production areas. Then, they were dried at low temperature, nucleated, and crushed through a sieve (60mesh), dried, and stored under constant weight for testing. The botanical origins of the samples were identified by Chengwu Fang. Voucher specimens have been deposited in the Chinese Medicine Resource Centre, Anhui University of
Chinese Medicine (Anhui, China). Information on the samples collected is listed in Table 1.

2.2. Preparation of Standard Solutions. Thirty standard substances were prepared by dissolving in ultrapure water, and their concentrations (in $\mathrm{mg} / \mathrm{mL}$ ) were as follows: (1) 0.17 , (2) 0.62 , (3) 0.15 , (4) 0.22 , (5) 0.12 , (6) 0.10 , (7) 0.25 , (8) 0.15 , (9) 0.40, (10) 0.36 , (11) 0.33 , (12) 4.2 , (13) 1.84 , (14) 0.36 , (15) $0.14,(16) 0.40,(17) 0.45$, (18) 0.16, , (19) 0.10, (20) 0.15 , (21) 1.5, , (22) $0.50,(23) 0.11$, (24) 0.21, (25) 0.10 , (26) 0.20, (27) $0.12,(28) 0.20,(29) 0.50$, and (30) 0.13. Mixed standard stock solution containing 30 standard substances was serially diluted with ultrapure water to the required concentration for establishment of calibration curves. All solutions were stored at $4^{\circ} \mathrm{C}$ and then passed through a $0.22 \mu \mathrm{m}$ membrane. The typical chromatograms of analytes and the sample are presented in Figure 1.

\subsection{Preparation of Sample Solutions. Accurately weighed} powder $(1.0 \mathrm{~g})$ was extracted by ultrasonication with $20 \mathrm{~mL}$ of ultrapure water for $30 \mathrm{~min}$ and cooled at normal temperature. The same solution was used to replenish the extraction system upon solvent loss due to volatilisation. The mixture was centrifuged at 10,000 rpm for $5 \mathrm{~min}$ and passed through the $0.22 \mu \mathrm{m}$ membrane before analyses.

2.4. UFLC-MS/MS Instrumentation and Conditions. Samples were analyzed using an UFLC system (Shimadzu, Kyoto, Japan) with a triple quadrupole-linear ion trap mass spectrometer (QUAD-4500; AB Sciex, Framingham, MA, USA). An XBridge Amide column $(100 \mathrm{~mm} \times 2.1 \mathrm{~mm}$, $3.5 \mu \mathrm{m}$; Waters, Milford, MA, USA) was used for chromatographic isolation. The mobile phase consisted of $0.2 \%$ aqueous formic acid (A) and $0.2 \%$ formic acid acetonitrile (B) at a velocity of $0.2 \mathrm{~mL} / \mathrm{min}$. Gradient elution was 0-2.5 min: $15 \%$ A; $2.5-5$ min: $15-50 \%$ A; 5-7 min: $50 \%$ A; 7-8 min: $50-15 \% \mathrm{~A}$; and $8-11 \mathrm{~min}: 15 \% \mathrm{~A}$. The column temperature was maintained at $30^{\circ} \mathrm{C}$, and the injection volume was $1 \mu \mathrm{L}$.

The ESI-MS spectra were acquired in the positive ion multiple reaction monitoring (MRM) mode with the nebuliser pressure $5.5 \mathrm{kV}$; gas temperature $550^{\circ} \mathrm{C}$; curtaingas pressure $241.3 \mathrm{kPa}$ (35 psi); gas-1 pressure $379.2 \mathrm{kPa}$ (55 psi); and gas-2 pressure $379.2 \mathrm{kPa}$ (55 psi). Simultaneously, detection of ion pairs, cluster voltage (DP), and collision voltage (CE) was optimized. All MS data were analyzed by Analyst 1.6.2 (AB SCIEX). The optimized parameters for MS for the 30 target components are shown in Table 2.

2.5. Method Validation. The proposed method was validated, including linearity, range, the limit of detection (LOD), the limit of quantitation (LOQ), precision, repeatability, stability, and recovery. Under the present chromatographic conditions, the LODs and LOQs were obtained on the response of each regression equation at signal-tonoise ratios $(\mathrm{S} / \mathrm{N})$ of 3 and 10 , respectively. The intra- and 
TABLE 1: The information of the sample from different regions.

\begin{tabular}{lcc}
\hline $\begin{array}{l}\text { Sample } \\
\text { number }\end{array}$ & $\begin{array}{c}\text { Cultivated } \\
\text { varieties }\end{array}$ & $\begin{array}{c}\text { Cultivation region } \\
\text { S1 }\end{array}$ \\
Daqingmei & $\begin{array}{c}\text { Shangyu, Shaoxing, } \\
\text { Zhejiang } \\
\text { Shangyu, Shaoxing, } \\
\text { Zhejiang }\end{array}$ \\
S3 & Daqingmei & Keqiao, Shaoxing, Zhejiang \\
S4 & Daqingmei & Lishui, Nanjing, Jiangsu \\
S5 & Nangao & Xishan, Suzhou, Jiangsu \\
S6 & Nangao & Yixing, Wuxi, Jiangsu \\
S7 & Nangao & Dayi, Chengdu, Sichuan \\
S8 & Shishengmei & Pingwu, Mianyang, Sichuan \\
S9 & Shishengmei & Baoxing, Yaan, Sichuan \\
S10 & Shishengmei & Eryuan, Dali, Yunnan \\
S11 & Yanmei & Eryuan, Dali, Yunnan \\
S12 & Yanmei & Eryuan, Dali, Yunnan \\
S13 & Yanmei & Guhe, Lijiang, Yunnan \\
S14 & Zhaoshuimei & Guhe, Lijiang, Yunnan \\
S15 & Zhaoshuimei & Guhe, Lijiang, Yunnan \\
S16 & Zhaoshuimei & Zhaoan, Zhangzhou, Fujian \\
S17 & Baimei & Zhaoan, Zhangzhou, Fujian \\
S18 & Baimei & Yongtai, Fuzhou, Fujian \\
\hline
\end{tabular}

interday precision were investigated by analysing the 30 analytes in six replicates within one and consecutive three days. The repeatability was ensured by preparing and analysing six independent sample solutions from sample S10. The stability of the samples was verified by analysing solution of sample S10 at 0,2, 4, 8, 12, and $24 \mathrm{~h}$. All variations were expressed by the relative standard deviation (RSD). The recovery test was performed to evaluate the accuracy of the above methods. The amount of 30 standards was added to six accurately weighed samples of $S 10(1.0 \mathrm{~g})$ and then extracted, processed, and quantified orderly, and the average recovery rate and RSD were calculated.

2.6. Multivariate Statistical Analyses. Principal component analysis (PCA) is used to visualize the similarity or difference in multivariate data. It is a method of transferring multiple variables through linear transformation to select fewer important variables. SPSS23.0 was used to evaluate the variation of 30 components in MF samples. OPLS-DA supervised by SIMCA-p 14.1 was conducted with data of 30 analytes to discover different chemical compositions of each sample.

TOPSIS is used commonly in analyses of multiple-objective decisions. It ranks a limited number of evaluation objects according to their proximity to the ideal target. By calculating the best and worst indices of samples, we introduced a method to evaluate the quality of samples based on the content of 30 analytes.

The data matrices of 21 amino acids and 9 nucleoside components in samples were normalized, and the corresponding matrices were established to calculate the $Z_{i j}$ value:

$$
Z_{i j}=\frac{X_{i j}}{\sqrt{\sum_{i=1}^{n} X_{i j^{2}}}},
$$

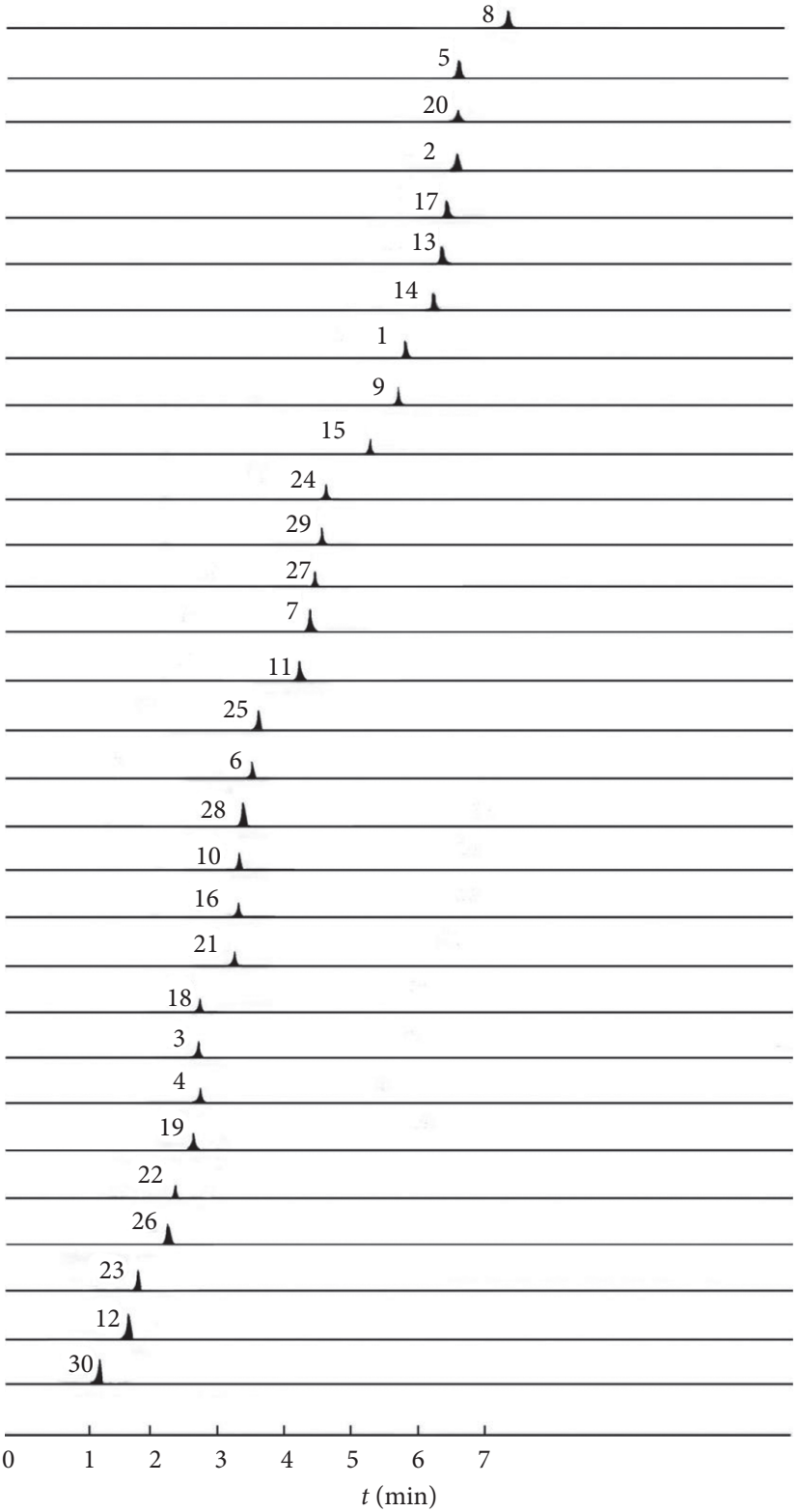

FIgURE 1: Multiple reaction monitoring (MRM) chromatograms of 30 compounds.

where $X_{i j}$ represents the value of the $i$ evaluation object on the $j$ index and $Z_{i j}$ represents the value of the $i$ evaluation object after normalization on the $j$ index.

The optimal vector $\left(Z^{+}\right)$and the worst vector $\left(Z^{-}\right)$consist of the maximum value and the minimum value of each column element. The distance from each evaluation object to $Z^{+}$and $Z^{-}$is calculated. $D_{i}^{+}$and $D_{i}^{-}$represent the distances of each evaluation object from $Z^{+}$and $Z^{-}$, respectively:

$$
\begin{aligned}
& D_{i}^{+}=\sqrt{\sum_{j=1}^{n}\left(z_{i j}-z_{j}^{+}\right)^{2}}, \\
& D_{i}^{-}=\sqrt{\sum_{j=1}^{n}\left(z_{i j}-z_{j}^{-}\right)^{2}} .
\end{aligned}
$$


TABLE 2: Optimised mass spectrometry parameters for determination of 30 components.

\begin{tabular}{|c|c|c|c|c|c|c|c|}
\hline Name & CAS no. & Formula & $t_{R}(\min )$ & {$[\mathrm{M}+\mathrm{H}]^{+}(\mathrm{m} / \mathrm{z})$} & MRM (precursor $\longrightarrow$ product) & $\mathrm{DP}(\mathrm{V})$ & $\mathrm{CE}(\mathrm{eV})$ \\
\hline$L$-aspartic acid & $56-84-8$ & $\mathrm{C}_{4} \mathrm{H}_{7} \mathrm{NO}_{4}$ & 1.70 & 134.05 & $134.05 / 87.96$ & 59 & 10 \\
\hline$L$-asparagine & $70-47-3$ & $\mathrm{C}_{4} \mathrm{H}_{8} \mathrm{~N}_{2} \mathrm{O}_{3}$ & 6.22 & 132.80 & $132.80 / 115.70$ & 46 & 13 \\
\hline$L$-serine & $56-45-1$ & $\mathrm{C}_{3} \mathrm{H}_{7} \mathrm{NO}_{3}$ & 6.12 & 106.05 & $106.05 / 59.99^{\mathrm{a}}$ & 67 & 8 \\
\hline Glycine & $56-40-6$ & $\mathrm{C}_{2} \mathrm{H}_{5} \mathrm{NO}_{2}$ & 5.34 & 76.04 & $76.04 / 30.00$ & 73 & 6 \\
\hline$L$-alanine & $56-41-7$ & $\mathrm{C}_{3} \mathrm{H}_{7} \mathrm{NO}_{2}$ & 4.39 & 90.06 & $90.06 / 44.02$ & 79 & 10 \\
\hline$L$-glutamic acid & $56-86-0$ & $\mathrm{C}_{5} \mathrm{H}_{9} \mathrm{NO}_{4}$ & 6.53 & 147.08 & $147.08 / 83.92$ & 83 & 14 \\
\hline$L$-valine & $72-18-4$ & $\mathrm{C}_{5} \mathrm{H}_{11} \mathrm{NO}_{2}$ & 3.38 & 118.09 & $118.09 / 72.06$ & 54 & 10 \\
\hline$L$-methionine & $63-68-3$ & $\mathrm{C}_{5} \mathrm{H}_{11} \mathrm{NO}_{2} \mathrm{~S}$ & 3.41 & 150.06 & $150.06 / 104.03$ & 91 & 10 \\
\hline$L$-isoleucine & $73-32-5$ & $\mathrm{C}_{6} \mathrm{H}_{13} \mathrm{NO}_{2}$ & 5.81 & 132.00 & $132.00 / 86.00$ & 66 & 15 \\
\hline$L$-histidine & $71-00-1$ & $\mathrm{C}_{6} \mathrm{H}_{9} \mathrm{~N}_{3} \mathrm{O}_{2}$ & 6.52 & 156.08 & $156.08 / 110.03$ & 95 & 16 \\
\hline$L$-arginine & $74-79-3$ & $\mathrm{C}_{6} \mathrm{H}_{14} \mathrm{~N}_{4} \mathrm{O}_{2}$ & 6.46 & 175.12 & $175.12 / 70.02$ & 88 & 18 \\
\hline$L$-threonine & $72-19-5$ & $\mathrm{C}_{4} \mathrm{H}_{9} \mathrm{NO}_{3}$ & 5.70 & 120.30 & $120.30 / 76.80$ & 54 & 11 \\
\hline$L$-leucine & $61-90-5$ & $\mathrm{C}_{6} \mathrm{H}_{13} \mathrm{NO}_{2}$ & 2.81 & 132.10 & $132.10 / 86.05$ & 98 & 10 \\
\hline$L$-phenylalanine & $63-91-2$ & $\mathrm{C}_{9} \mathrm{H}_{11} \mathrm{NO}_{2}$ & 2.84 & 166.10 & $166.10 / 120.05$ & 56 & 14 \\
\hline$L$-cystine & $56-89-3$ & $\mathrm{C}_{6} \mathrm{H}_{12} \mathrm{~N}_{2} \mathrm{O}_{4} \mathrm{~S}_{2}$ & 7.02 & 240.80 & $240.80 / 151.90$ & 71 & 18 \\
\hline$L$-tyrosine & $60-18-4$ & $\mathrm{C}_{9} \mathrm{H}_{11} \mathrm{NO}_{3}$ & 3.56 & 182.16 & $182.16 / 136.08$ & 46 & 17 \\
\hline$L$-hydroxyproline & $51-35-4$ & $\mathrm{C}_{5} \mathrm{H}_{9} \mathrm{NO}_{3}$ & 2.89 & 133.80 & $133.80 / 71.80$ & 52 & 25 \\
\hline$L$-proline & $147-85-3$ & $\mathrm{C}_{5} \mathrm{H}_{9} \mathrm{NO}_{2}$ & 4.13 & 116.07 & $116.07 / 70.02$ & 68 & 10 \\
\hline$L$-tryptophan & $73-22-3$ & $\mathrm{C}_{11} \mathrm{H}_{12} \mathrm{~N}_{2} \mathrm{O}_{2}$ & 2.73 & 205.00 & $205.00 / 188.00$ & 202 & 15 \\
\hline$\gamma$-Aminobutyric acid & $56-12-2$ & $\mathrm{C}_{4} \mathrm{H}_{9} \mathrm{NO}_{2}$ & 3.14 & 103.70 & $103.70 / 86.90$ & 32 & 14 \\
\hline$L$-lysine hydrochloride & $10098-89-2$ & $\mathrm{C}_{6} \mathrm{H}_{15} \mathrm{CIN}_{2} \mathrm{O}_{2}$ & 6.52 & 147.00 & $147.00 / 84.00$ & 52 & 24 \\
\hline Uracil & $66-22-8$ & $\mathrm{C}_{4} \mathrm{H}_{4} \mathrm{~N}_{2} \mathrm{O}_{2}$ & 2.44 & 113.04 & $113.04 / 70.00$ & 111 & 21 \\
\hline Adenine & $73-24-5$ & $\mathrm{C}_{5} \mathrm{H}_{5} \mathrm{~N}_{5}$ & 1.93 & 136.06 & $136.06 / 136.06$ & 51 & 24 \\
\hline Cytidine & $65-46-3$ & $\mathrm{C}_{9} \mathrm{H}_{13} \mathrm{~N}_{3} \mathrm{O}_{5}$ & 4.77 & 244.09 & $244.09 / 112.00$ & 61 & 10 \\
\hline Inosine & $58-63-9$ & $\mathrm{C}_{10} \mathrm{H}_{12} \mathrm{~N}_{4} \mathrm{O}_{5}$ & 3.66 & 269.00 & $269.00 / 137.05$ & 46 & 15 \\
\hline Vernine & $118-00-3$ & $\mathrm{C}_{10} \mathrm{H}_{13} \mathrm{~N}_{5} \mathrm{O}_{5}$ & 4.56 & 284.30 & $284.30 / 152.00$ & 62 & 15 \\
\hline Thymidine & $50-89-5$ & $\mathrm{C}_{10} \mathrm{H}_{14} \mathrm{~N}_{2} \mathrm{O}_{5}$ & 2.39 & 243.10 & $243.10 / 127.07$ & 61 & 13 \\
\hline Adenosine & $58-61-7$ & $\mathrm{C}_{10} \mathrm{H}_{13} \mathrm{~N}_{5} \mathrm{O}_{4}$ & 3.47 & 268.10 & $268.10 / 136.07$ & 86 & 23 \\
\hline Uridine & $58-96-8$ & $\mathrm{C}_{9} \mathrm{H}_{12} \mathrm{~N}_{2} \mathrm{O}_{6}$ & 4.72 & 244.90 & $244.90 / 113.00$ & 103 & 13 \\
\hline Hypoxanthine & $68-94-0$ & $\mathrm{C}_{5} \mathrm{H}_{4} \mathrm{~N}_{4} \mathrm{O}$ & 1.15 & 137.05 & $137.05 / 137.05$ & 51 & 24 \\
\hline
\end{tabular}

The relative closeness to the ideal object $\left(C_{i}\right)$ is calculated, and $C_{i}$ values are compared to rank alternatives. When $C_{i}$ is between 0 and 1 , closer to 1 , the evaluation object is close to the optimal level; when it is closer to 0 , the evaluation object is close to the worst level:

$$
C_{i}=\frac{D_{i}^{-}}{D_{i}^{-}+D_{i}^{+}} .
$$

\section{Results and Discussion}

3.1. Sample Preparation Optimization. In order to obtain quantitative results, the extraction method was to be optimized. The extraction method adopted ultrasound extraction with water according to [26]. And the solvent volume $(10,20,30$, and $40 \mathrm{~mL})$ and extraction time $(10,20,30$, and $40 \mathrm{~min}$ ) were examined. The results showed that the optimal extraction method was ultrasonic treatment at room temperature for $30 \mathrm{~min}$ with $20 \mathrm{~mL}$ water as the solvent according to characteristic peak intensity.

3.2. Optimization of UFLC-MS/MS Conditions. Previous studies $[16,17]$ reported that the positive ion modes presented higher sensitivity and intensity in examining components of amino acids and nucleosides than negative ion modes, so the ESI+ mode was adopted in the study. Most components obtained intensive product ions by optimization of the MS parameters; just adenine and hypoxanthine were too low to be detected.

Chromatographic conditions were optimized. Different mobile phases (water/methanol; $0.1 \%$ aqueous formic acid/ methanol; $0.1 \%$ aqueous formic acid/acetonitrile; and $0.2 \%$ aqueous formic acid/0.2\% formic acid acetonitrile) were investigated. We found that acetonitrile had better separation efficiency than that of methanol. Upon addition of $0.2 \%$ aqueous formic acid into acetonitrile and water, the shape of the chromatographic peak and degree of separation were better, and the tail of the chromatographic peak was reduced. Furthermore, a flow rate of $0.1-0.5 \mathrm{~mL} / \mathrm{min}$, injection volume of $1-5 \mu \mathrm{L}$, and column temperature of $30-40^{\circ} \mathrm{C}$ were studied to obtain rapid and reliable separation. As a result, the analysis was carried out on a Waters XBridge Amide column $(100 \mathrm{~mm} \times 2.1 \mathrm{~mm}, 3.5 \mu \mathrm{m})$ with elution by mobile phase of $0.2 \%$ aqueous formic acid (A) and $0.2 \%$ formic acid acetonitrile (B) at a flow rate of $0.2 \mathrm{~mL} / \mathrm{min}$ with $1 \mu \mathrm{L}$ per injection. The column temperature was maintained at $30^{\circ} \mathrm{C}$, respectively.

3.3. Method Validation. Validations of the method are shown in Table 3. All calibration curves showed good linearity regressions $\left(R^{2}>0.9990\right)$ within the determination range. The LODs and LOQs of the 30 compounds were estimated to be in the range of $0.14-5.00 \mathrm{ng} / \mathrm{mL}$ and $0.18-16.67 \mathrm{ng} / \mathrm{mL}$, respectively. They should provide 
TABLE 3: Regression equations, detection limits (LOD), quantitation limits (LOQ), intraday and diurnal precision, stability, repeatability, recovery, and matrix effects of 30 components.

\begin{tabular}{|c|c|c|c|c|c|c|c|c|c|c|c|c|}
\hline No. & Regression equation & $\begin{array}{l}\text { Linear range } \\
(\mu \mathrm{g} / \mathrm{mL})\end{array}$ & $R^{2}$ & $\begin{array}{l}\text { LoD } \\
(\mathrm{ng} / \\
\mathrm{mL}) \\
\end{array}$ & $\begin{array}{l}\text { LoQ } \\
\text { (ng/ } \\
\text { mL) }\end{array}$ & $\begin{array}{c}\text { Precision } \\
\text { Intraday } \\
(n=6)\end{array}$ & $\begin{array}{l}(\mathrm{RSD} \%) \\
\text { Interday } \\
(n=3)\end{array}$ & $\begin{array}{c}\text { Stability } \\
\text { (RSD\%, } \\
n=6)\end{array}$ & $\begin{array}{c}\text { Repeatability } \\
\text { (RSD\%, } \\
n=6)\end{array}$ & $\begin{array}{l}\text { Reco } \\
\text { Mean }\end{array}$ & $\begin{array}{l}\text { very } \\
\text { RSD } \\
\% \\
\end{array}$ & $\begin{array}{c}\text { Matrix } \\
\text { effect }\end{array}$ \\
\hline 1 & $y=9421.6 x+136392$ & $0.172-17.23$ & 0.9997 & 0.18 & 0.59 & 2.32 & 2.31 & 2.83 & 2.58 & 104.38 & 3.34 & 1.01 \\
\hline 2 & $y=810.83 x+56376$ & $0.625-62.51$ & 0.9995 & 0.78 & 2.6 & 2.32 & 2.26 & 2.81 & 2.45 & 95.54 & 2.37 & 1.03 \\
\hline 3 & $y=26896 x+995235$ & $0.157-15.72$ & 0.9996 & 5.00 & 16.67 & 2.17 & 2.79 & 2.22 & 2.21 & 97.39 & 3.69 & 1.05 \\
\hline 4 & $y=24.819 x+221136$ & $0.301-22.89$ & 0.9994 & 2.73 & 9.00 & 1.62 & 2.89 & 2.39 & 2.73 & 104.39 & 2.18 & 1.02 \\
\hline 5 & $y=3025.2 x+667487$ & $0.0623-12.47$ & 0.9993 & 0.14 & 0.18 & & 2.38 & 2.87 & & 101.54 & 3.55 & 0.96 \\
\hline 6 & $y=116421 x-47671$ & $0.101-10.13$ & 0.9998 & 0.16 & 0.54 & 1.99 & 2.77 & 1.75 & 2.68 & 96.56 & 2.65 & 0.99 \\
\hline 7 & $y=472881 x-302217$ & $0.156-25.98$ & 0.9993 & 4.69 & 15.63 & 2.31 & 1.99 & 2.61 & 2.21 & 99.61 & 2.01 & 1.03 \\
\hline 8 & $y=61.23 x+5234.1$ & $0.00153-15.38$ & 0.9997 & 0.16 & 0.55 & 3.29 & 2.68 & 2.51 & 2.89 & 97.75 & 2.44 & 0.97 \\
\hline 9 & $y=3025.2 x+667384$ & $0.203-40.69$ & 0.9998 & 2.73 & 9.09 & 2.33 & 2.38 & 2.92 & 2.49 & 98.33 & 3.03 & 0.97 \\
\hline 10 & $y=15.908 x-156.29$ & $0.306-36.75$ & 0.9998 & 1.28 & 4.27 & 2.79 & 1.78 & 1.44 & & & 2.29 & 0.97 \\
\hline 11 & $y=18.312 x+146822$ & $0.301-33.16$ & 0.9999 & 0.51 & 1.69 & 2.35 & 2.41 & 2.27 & 2.23 & 102.31 & 2.59 & 1.01 \\
\hline 12 & $y=484405 x-927738$ & $4.211-420.13$ & 0.9992 & 2.75 & 9.17 & 1.28 & 2.31 & 1.86 & 2.68 & 95.02 & 2.18 & 0.95 \\
\hline 13 & $y=431347 x-145124$ & $1.847-184.73$ & 0.9994 & 0.37 & 1.23 & 1.56 & 2.01 & 2.46 & 1.74 & 97.35 & 2.56 & 0.98 \\
\hline 14 & $y=242.73 x+45133$ & $0.919-36.75$ & 0.9996 & 1.85 & 6.17 & & & & & 100.57 & 2.67 & 1.02 \\
\hline 15 & $y=438305 x-224115$ & $0.919-14.19$ & 0.9998 & 3.00 & 10.00 & 2.38 & 2.12 & 2.79 & 2.95 & 96.52 & 2.82 & 0.99 \\
\hline 16 & $y=2745.6 x-132315$ & $1.617-40.43$ & 0.9998 & 0.50 & 1.66 & 2.06 & 2.68 & 1.82 & 2.75 & 97.41 & 2.91 & 0.94 \\
\hline 17 & $y=669.72 x+38649$ & $2.281-45.62$ & 0.9997 & 2.00 & 0.67 & & 2.59 & 2.12 & & 102.23 & 2.61 & 0.96 \\
\hline 18 & $y=107.22 x+129047$ & $0.0169-16.94$ & 0.9996 & 0.22 & 0.73 & 2.21 & 2.3 & & 2.8 & 98.03 & 3.89 & 0.98 \\
\hline 19 & $y=102.67 x+2102.4$ & $0.0121-10.21$ & 0.9996 & 0.83 & 2.78 & 2.73 & 2.56 & 2.09 & 2.88 & 98.35 & 2.61 & 0.96 \\
\hline 20 & $y=5894.2 x+683598$ & $0.784-15.68$ & 0.9998 & 3.26 & 10.9 & 2.28 & 2.36 & 2.59 & 2.66 & 96.73 & 2.67 & 1.01 \\
\hline 21 & $y=1289.2 x+364183$ & $0.503-150.97$ & 0.9992 & 0.4 & 1.33 & 2.06 & 1.99 & 2.16 & 2.01 & 104.94 & 2.09 & 0.98 \\
\hline 22 & $y=294.16 x+20062$ & $0.0559-50.27$ & 0.9997 & 1.47 & 4.90 & & 2.56 & 2.17 & & 102.21 & 2.88 & 1.01 \\
\hline 23 & $y=34948 x-92208$ & $0.168-11.68$ & 0.9997 & 0.88 & 2.94 & 1.99 & 2.37 & 2.89 & 2.25 & 97.15 & 2.31 & 1.05 \\
\hline 24 & $y=5235.4 x+8471.5$ & $0.0287-20.87$ & 0.9996 & 0.16 & 0.54 & 2.14 & 2.78 & 2.03 & 2.01 & 98.45 & 2.51 & 0.97 \\
\hline 25 & $y=9189.8 x-69366$ & $0.0534-10.68$ & 0.9995 & 0.63 & 2.08 & 1.75 & 2.88 & 2.33 & 2.07 & 96.64 & 2.44 & 0.98 \\
\hline 26 & $y=282.98 x+42660$ & $0.0102-20.43$ & 0.9997 & 3.37 & 11.2 & 1.38 & 2.13 & 2.26 & 2.28 & 94.89 & 2.11 & 0.93 \\
\hline 27 & $y=10624 x+38109$ & $0.0127-12.76$ & 0.9998 & 0.80 & 2.67 & 2.38 & 2.34 & 2.86 & 2.46 & 103.21 & 2.61 & 1.03 \\
\hline 28 & $y=13059 x-240433$ & $0.205-20.51$ & 0.9991 & 0.66 & 2.18 & 1.91 & 3.23 & 1.52 & 2.07 & 99.18 & 2.47 & 0.94 \\
\hline 29 & $y=32.836 x+2686.9$ & $0.145-30.97$ & 0.9995 & 2.11 & 7.04 & 1.85 & 2.13 & 2.76 & 1.94 & 97.26 & 2.06 & 1.03 \\
\hline 30 & $y=22649 x+62656$ & $0.0106-13.79$ & 0.9991 & 0.22 & 1.23 & 2.06 & 1.97 & 2.23 & 2.06 & 103.24 & 2.36 & 1.02 \\
\hline
\end{tabular}

satisfactory sensitivity for all analytes. The RSD values of intraday, interday, repeatability, and stability tests of the 30 compounds were $1.28 \%-3.29 \%, 1.78-3.23 \%, 1.74 \%-2.95 \%$, and $1.44 \%-2.92 \%$, respectively. The average recovery rates were $95.02-104.94 \%$, with the RSD values of $2.01-3.89 \%$, and the slope ratio of the matrix curve to the pure solution curve was $0.93-1.05$. The results showed the established method could provide sufficient accuracy and stability for the qualitative and quantitative determination of MF samples.

In order to highlight the sensitivity of the method, the LODs of each target compound were compared with other techniques: MLC-HSLC [27], HILIC-UHPLC-QTRAP/MS [28], and UPLC-MS/MS [16], and the results of comparison are listed in Table S1. The LOD range of our method is $0.14-5.00 \mathrm{ng} / \mathrm{mL}$, and the LOD range of other methods is $250-410 \mathrm{ng} / \mathrm{mL}, \quad 0.45-53.5 \mathrm{ng} / \mathrm{mL}$, and $0.17-61.2 \mathrm{ng} / \mathrm{mL}$, respectively. The LOD range of nucleoside components was 0.16-3.37 ng/mL, although few nucleoside components in other methods were available for effective comparison, but overall, this method was more sensitive than other methods, especially in arginine, alanine, and asparagine. The previous studies used the amino acid analyzer and PITC-HPLC methods [20, 21] in MF. However, both methods showed some disadvantages in resolution and analysis time, required over 90 minutes. Our solution time was 11 min included balanced column conditions, which means this analytical method should take shorter time than other methods in MF.

3.4. Quantitative Analyses. Contents of the 30 components in samples were determined using UFLC-MS/MS. Quantitative determination is shown in Table S2. The data from all the samples elucidated that the total contents of amino acids and nucleosides were significantly different between varieties of MF in the order Nangao $(123800.00 \mu \mathrm{g} / \mathrm{g})$ $>$ Daqingmei $(8330.99 \mu \mathrm{g} / \mathrm{g})>$ Zhaoshuimei $(8081.55 \mu \mathrm{g} / \mathrm{g})$ $>$ Yanmei $\quad(6870.08 \mu \mathrm{g} / \mathrm{g})>$ Shishengmei $\quad(4622.85 \mu \mathrm{g} / \mathrm{g})$ $>$ Baimei $(2599.75 \mu \mathrm{g} / \mathrm{g})$.

In terms of individual components, aspartic acid is the most abundant amino acid in MF, which is consistent with the previous results [2]. In all the samples, the average of the aspartic acid content was $2599.41 \mu \mathrm{g} / \mathrm{g}$ followed by $L$-asparagine, $\gamma$-aminobutyric acid, $L$-valine, and $L$-histidine; thymidine was the lowest $(0.54 \mu \mathrm{g} / \mathrm{g})$. Adenosine had the highest content among the nucleoside components followed by uridine uracil, and adenine. Interestingly, the content of aspartic acid in the samples from Nangao was significantly higher than the other varieties. Aspartic acid can regulate the 

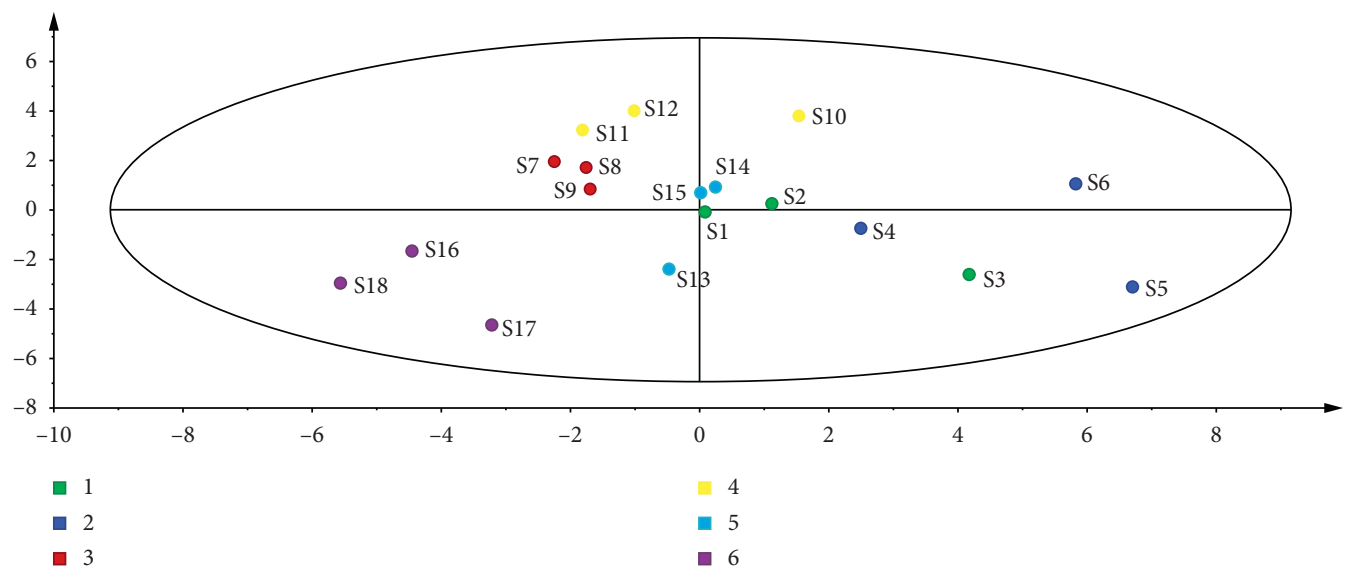

(a)

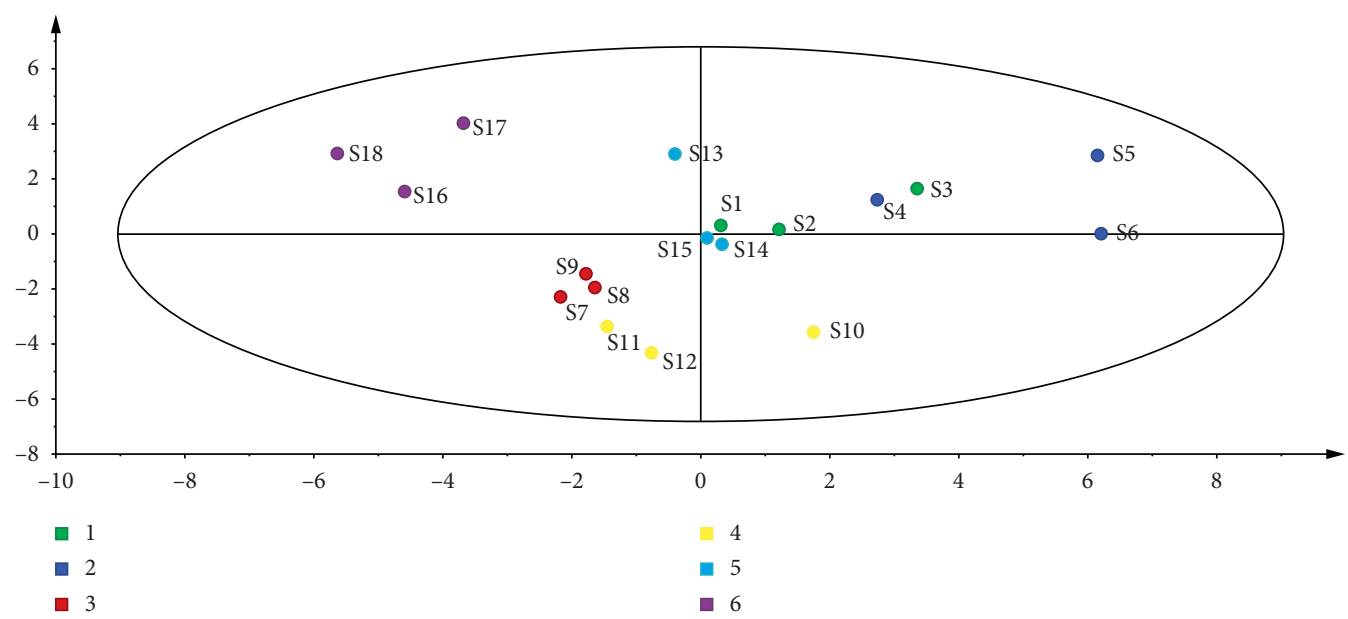

(b)

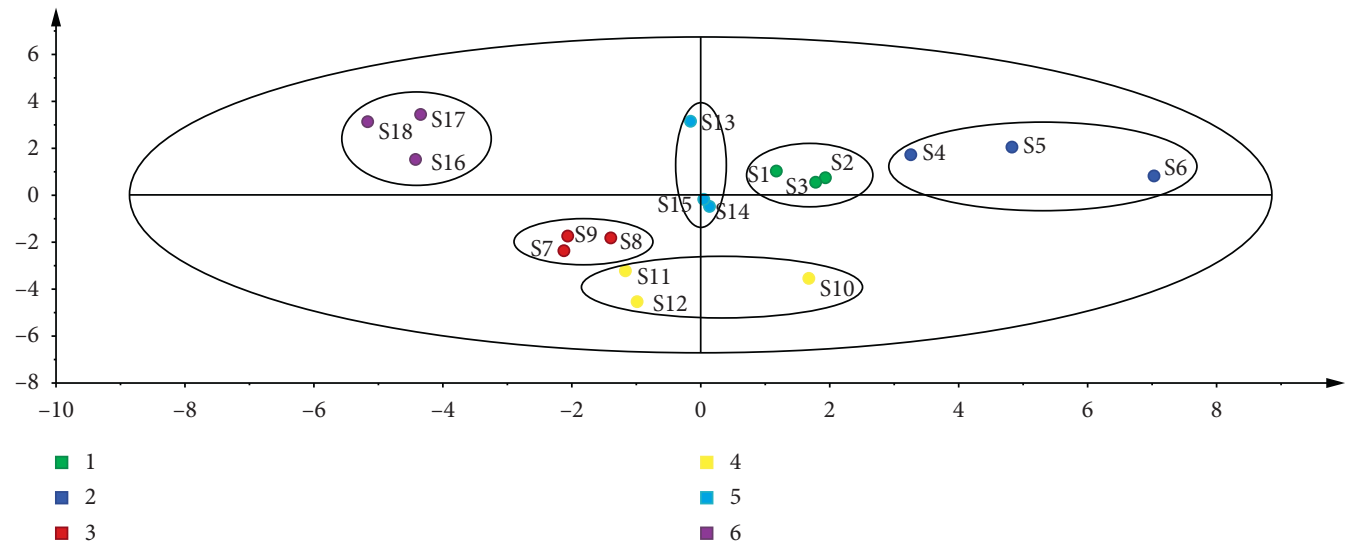

(c)

Figure 2: Continued. 


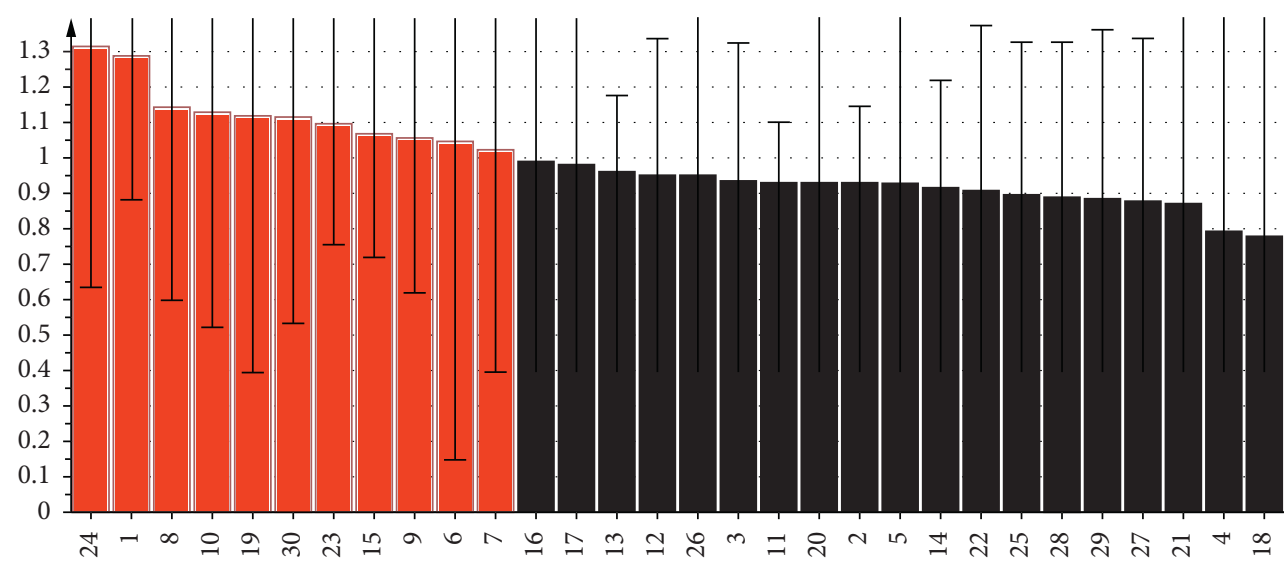

(d)

FIgURe 2: Multivariate statistical analyses of samples. (a) Principal component analysis (PCA) score chart. (b) Partial least square discriminant analysis (PLS-DA) score chart. (c) Orthogonal partial least square discriminant analysis (OPLS-DA) score chart. (d) VIP.

metabolism of the brain and nerves and is often used to treat heart disease, liver dysfunction, and hypertension [13, 14]. Adenosine, as an antiarrhythmic drug, can treat angina pectoris, myocardial infarction, cerebrovascular disorders, etc. Therefore, we speculated that the clinical, medicinal, and edible value of Nangao was higher than the other varieties.

3.5. Multivariate Statistical Analyses of Samples. Analyzed data for 30 compositions of each sample are shown in Figure 2. After the original data were standardized, PCA was performed with SPSS23.0 to obtain the eigenvalues and variance contributions of principle components (PCs) $[29,30]$. The results are given in Table S3. The eigenvalues of the first seven PCs were greater than 1, the cumulative contributions reached $89.04 \%$, and the first three PCs (35.89\% for PC1, 20.88\% for PC2, and $11.68 \%$ for PC3) simplified the multidimensional dataset to a $3 \mathrm{D}$ dataset; the remaining PCs were discarded for the less effect. PC1 had good correlation with $L$-aspartic acid, $L$-asparagine, $L$-histidine, and $L$-proline. PC2 showed good correlation with $L$ valine, adenine, and hypoxanthine, and PC3 showed good correlation with glycine (Table S4). The results indicated that the aforementioned components might contribute to the classification of samples. According to the scatter plots (Figure 2(a)), PCA results showed the differences between samples but could not provide a clear classification for the samples.

PLS-DA and OPLS-DA were used to extend to discover the factors leading to different varieties [31, 32]; two methods were selected for this study. The PLS-DA results showed R2Y was 0.883 , indicating that the model fitted well, but the model according to cross validation $Q 2=0.44<0.5$ indicated that the model prediction result was not ideal; the score plot is shown in Figure 2(b). The OPLS-DA results showed R2Y was 0.878 , indicating that the model fitted well, and $Q 2=0.525>0.5$ indicated that the model predicted well. In the OPLS-DA score plot (Figure 2(c)), the samples were clearly divided into six groups; each group corresponded to the varieties of samples. In the VIP loading diagram of
TABLE 4: Sequencing of samples according to quality by TOPSIS.

\begin{tabular}{lcccc}
\hline Sample & $D_{i}^{+}$ & $D_{i}^{-}$ & $C_{i}$ & Ranking \\
\hline S1 & 2.1051 & 0.9899 & 0.3198 & 10 \\
S2 & 1.7748 & 1.4024 & 0.4414 & 4 \\
S3 & 1.8297 & 1.5782 & 0.4631 & 3 \\
S4 & 1.8716 & 1.1848 & 0.3876 & 5 \\
S5 & 1.7277 & 1.7495 & 0.5031 & 1 \\
S6 & 1.7289 & 1.6868 & 0.4938 & 2 \\
S7 & 2.2416 & 0.9874 & 0.3058 & 11 \\
S8 & 2.3036 & 0.6737 & 0.2263 & 17 \\
S9 & 2.1677 & 0.8285 & 0.2765 & 13 \\
S10 & 1.9502 & 1.1903 & 0.3790 & 7 \\
S11 & 2.3375 & 0.6425 & 0.2156 & 18 \\
S12 & 2.1381 & 1.1526 & 0.3503 & 9 \\
S13 & 1.9987 & 1.2252 & 0.3800 & 6 \\
S14 & 2.0088 & 1.0863 & 0.3510 & 8 \\
S15 & 2.1371 & 0.9396 & 0.3054 & 12 \\
S16 & 2.4264 & 0.7281 & 0.2308 & 16 \\
S17 & 2.3657 & 0.8963 & 0.2748 & 14 \\
S18 & 2.4811 & 0.8541 & 0.2561 & 15 \\
\hline
\end{tabular}

OPLS-DA (Figure 2(d)), the large load (VIP > 1) could be regarded as a marker component that contributed greatly to the classification of samples; $L$-isoleucine, cytidine, $L$-cystine, $L$-methionine, glycine, hypoxanthine, $L$-tyrosine, adenine, $L$-arginine, $L$-alanine, and $L$-threonine could distinguish different varieties of MF samples. These results indicated that amino acids and nucleosides might be regarded as chemical markers for MF classification.

Samples were analyzed comprehensively by TOPSIS, and ranking results were obtained. The data matrix of 30 components in six varieties was normalized, and the corresponding standardized matrix was established. $Z_{i j}$ value was calculated according to formula (1), and the results are shown in Table S5. The optimal vector $\left(Z^{+}\right)$and the worst vector $\left(Z^{-}\right)$were obtained according to the normalized samples: $Z^{+}=(0.4509,0.4931,0.4299,0.4362,0.6519,0.5515$, 0.4801, 0.6745, 0.4068, 0.4464, 0.5485, 0.6494, 0.4531, 0.5864, $0.4075,0.4956,0.4481,0.4908,0.4650,0.5296,0.5622,0.4623$, $0.4171,0.4140,0.5792,0.9878,0.6561,0.5689,0.4469$, 
$0.4113) ; Z^{-}=(0.0222,0.0031,0.0225,0.0862,0.0304,0.0357$, 0.0475, 0.0000, 0.0502, 0.0451, 0.0000, 0.0110, 0.0350, 0.0000, 0.0169, 0.0000, 0.0463, 0.0500, 0.0000, 0.0000, 0.0509, 0.0857, $0.0864,0.0352,0.0074,0.0000,0.0244,0.0348,0.0760$, $0.1062)$.

$D_{i}^{+}, D_{i}^{-}$, and $C_{i}$ were calculated according to formulas (2) and (3), respectively, and the results are shown in Table 4. The evaluation objects were ranked according to $C_{i}$. The larger the value of $C_{i}$, the better the quality. We noted significant differences in the content between different varieties, the results above demonstrated that Nangao and Daqingmei were better qualities than other varieties of MF.

\section{Conclusion}

A rapid and sensitive UFLC-MS/MS method was established and employed to determine the components of 21 amino acids and 9 nucleosides in different varieties of $\mathrm{MF}$. According to the analytical results, MF had rich amino acids and nucleosides, and aspartic acid was the most abundant amino acid in MF followed by $L$-asparagine, $\gamma$-aminobutyric acid, $L$-valine, and $L$-histidine. Adenosine had the highest content among nucleoside components followed by uridine, uracil, and adenine. Six varieties were well classified by PCA and OPLS-DA; 11 amino acids and nucleoside components could be used as chemical markers to classify different varieties of MF. As medicinal and edible varieties, the content and quality of Nangao and Daqingmei were higher than other varieties; the obvious difference should be caused by the artificial breeding of varieties. The present study could provide theoretical basis for further evaluation of the internal quality and control of medicinal and edible MF.

\section{Data Availability}

The data used to support the findings of this study are available from the corresponding author upon request.

\section{Conflicts of Interest}

All authors declare that they have no conflicts of interest.

\section{Acknowledgments}

This research was supported by the Natural Science Foundation of Education Department of Anhui Province, China (KJ2019A0479), the Capacity Building Project for Major Increase or Decrease in Expenditures at the Central Level (2060302-1604-01), the Demonstration Funds for the Promotion of Forestry Science and Technology from the Central Finance (Z175070050002), and the Anhui Provincial Natural Science Foundation (2008085QH394).

\section{Supplementary Materials}

Figure S1: the chemical structures of 30 amino acid and nucleotide standard compounds. Table S1: the LODs detected by UFLC-MS/MS, MLC -HSLC, HILIC-UHPLCQTRAP/MS, and UPLC -MS/MS to analyze the sensitivity of four methods. Table S2: contents of 30 amino acids and nucleosides in samples by UFLC -MS/MS method. Table S3: eigenvalue and variance contributions of principle components (PCs). After standardizing the original data of 30 components, PCA was performed with SPSS23.0 to obtain the eigenvalues and variance contributions of PCs. Table S4: initial factor loading matrix of the first three PCs. Table S5: matrix after normalization of 30 components in samples. Using formula (1) to calculate $\mathrm{Zij}$ value.. (Supplementary Materials)

\section{References}

[1] Chinese Pharmacopoeia Commission, Pharmacopoeia of the People's Republic of China, vol. 1, China Medical Science and Technology Press, Beijing, China, 2015.

[2] H. Y. Zhang, Q. Li, and X. L. Fu, "Research progress on chemical component and pharmacological effects of Wumei (Mume fructus)," Shanghai Journal of Chinese Medicine, vol. 51, no. S1, pp. 296-300, 2017.

[3] Y. J. Kwon, D. Y. Kwon, and J. H. Lee, "Evaluation of antibacterial effects of a combination of Coptidis rhizoma, Mume fructus, and Schizandrae fructus against Salmonella," International Journal of Food Microbiology, vol. 127, no. 1-2, pp. 180-183, 2008.

[4] J. C. Park, J. H. Ma, W. K. Jeon, and J. S. Han, "Fructus mume extracts alleviate cognitive impairments in 5XFAD transgenic mice," BMC Complementary and Alternative Medicine, vol. 16, no. 1, pp. 54-65, 2015.

[5] T. Hoshino, H. Takagi, A. Naganuma et al., "Advanced hepatocellular carcinoma responds to MK615, a compound extract from the Japanese apricot "Prunus mume"," World Journal of Hepatolgy, vol. 5, no. 10, pp. 596-600, 2013.

[6] N. Sriwilaijaroen, A. Kadowaki, Y. Onishi et al., "Mumefural and related HMF derivatives from Japanese apricot fruit juice concentrate show multiple inhibitory effects on pandemic influenza A (H1N1) virus," Food Chemistry, vol. 127, no. 1, pp. 1-9, 2011.

[7] J. Y. Chen, Chinese Mei Flowers, Hainan Publishing House, Haikou, China, 1996, in Chinese.

[8] L. D. Sun, Y. Q. Wang, X. L. Yan et al., "Genetic control of juvenile growth and botanical architecture in an ornamental woody plant, Prunus mume Sieb. et Zucc. as revealed by a high-density linkage map," BMC Genetics, vol. 15, no. S1, 2014.

[9] Y. X. Liu, J. X. Yang, X. L. Xu, and Q. W. Li, "Quality characteristics, and antibacterial and antioxidant activity of Prunus mume fruits," Food Science, vol. 40, no. 1, pp. 77-84, 2019.

[10] M. Turturică, N. Stănciuc, G. Bahrim, and G. Râpeanu, “Effect of thermal treatment on phenolic compounds from plum (Prunus domestica) extracts-a kinetic study," Journal of Food Engineering, vol. 171, pp. 200-207, 2016.

[11] C. Ding and B. S. Li, "Changes of colors in Prunus mume during smoking processing," Modern Food Science and Technology, vol. 28, no. 1, pp. 23-26, 2012.

[12] N. Vale, A. Ferreira, J. Matos, P. Fresco, and M. Gouveia, "Amino acids in the development of prodrugs," Molecules, vol. 23, no. 9, pp. 1-61, 2018.

[13] O. Wahl and U. Holzgrabe, "Amino acid analysis for pharmacopoeial purposes," Talanta, vol. 154, pp. 150-163, 2016.

[14] G. Wu, "Functional amino acids in nutrition and health," Amino Acids, vol. 45, no. 3, pp. 407-411, 2013. 
[15] A. Khan, J. H. Pan, S. Cho, S. Lee, Y. J. Kim, and Y. H. Park, "Investigation of the hepatoprotective effect of Prunus mume Sieb. et Zucc extract in a mouse model of alcoholic liver injury through high-resolution metabolomics," Journal of Medicinal Food, vol. 20, no. 8, pp. 734-743, 2017.

[16] C. Qu, H. Yan, S. Q. Zhu et al., "Comparative analysis of nucleosides, nucleobases, and amino acids in different parts of Angelicae sinensis radix by ultra high performance liquid chromatography coupled to triple quadrupole tandem mass spectrometry," Journal of Separation Science, vol. 42, no. 6, pp. 1122-1132, 2019.

[17] Z. Cai, C. Wang, C. Chen et al., "Quality evaluation of Lonicerae japonicae flos and lonicerae flos based on simultaneous determination of multiple bioactive constituents combined with multivariate statistical analysis," Phytochemical Analysis, pp. 1-12, 2019.

[18] J. J. Li, A. Q. Zhao, D. M. Li, and Y. He, "Comparative study of the free amino acid compositions and contents in three different botanical origins of Coptis herb," Biochemical Systematics Ecology, vol. 83, pp. 117-120, 2019.

[19] Z. P. Liu, Y. Y. Guo, and J. J. Liu, "Effect of strain and site of Dendrobium candidum on amino acid content," China Journal of Traditional Chinese Medicine, vol. 40, no. 8, pp. 1468-1472, 2015.

[20] X. Y. Liu, B. Pu, and Y. Liu, "Determination and study of basic nutrient components in dayi fruit and plum," Food Research Develop, vol. 28, no. 6, pp. 146-148, 2007.

[21] J. H. Li, X. Lei, and T. Yang, "Determination of 17 free amino acids content in Wumei by PITC pre-column derivatization HPLC," Journal of Chinese Medicine Materials, vol. 42, no. 6, pp. 1334-1338, 2019.

[22] Z. Wu, H. Li, and Y. Yang, "Geographical origin traceability and varietal classification of Zanthoxylum based on mineral profile," Food Science, vol. 40, no. 16, pp. 213-219, 2019.

[23] Y. Y. Ba, R. Xiao, and Q. J. Chen, "Comprehensive quality evaluation of polygoni Orientalis fructus and its processed product: chemical fingerprinting and simultaneous determination of seven major components coupled with chemometric analyses," Phytochemical Analysis, 2019.

[24] B. Özkan, O. Dengiz, and I. D. Turan, "Site suitability assessment and mapping for rice cultivation using multi-criteria decision analysis based on fuzzy-AHP and TOPSIS approaches under semihumid ecological condition in delta plain," Paddy and Water Environment, vol. 17, no. 4, pp. 665-676, 2019.

[25] Y. Li, P. Hu, and Y. Q. Zhang, "Simultaneous determination of amino acid and nucleoside components in Cherimoya cherimoya from different habitats by (UPLC-MS/MS) and PCA and TOPSIS analysis," Chinese Traditional and Herbal Drugs, vol. 49, no. 11, pp. 2671-2677, 2018.

[26] Y. J. Zhang, P. Hu, Y. Ma et al., "Simultaneous determination of amino acids and nucleosides of three species in termitomyces by UFLC-QTRAP-MS-MS," China Journal of Chinese Materrial Medica, vol. 42, no. 6, pp. 1152-1159, 2017.

[27] Y. Xie, T. Luo, J. Yang, and Y. Dong, "Rapid determination of amino acids in beer, red wine, and donkey-hide gelatin by gradient elution of HPLC: from micellar liquid chromatography to high submicellar liquid chromatography," Journal of AOAC International, vol. 101, no. 1, pp. 249-255, 2018.

[28] G. Zhou, M. Wang, Y. Li, Y. Peng, and X. Li, "Rapid and sensitive analysis of 27 underivatized free amino acids, dipeptides, and tripeptides in fruits of Siraitia grosvenorii swingle using HILIC-UHPLC-QTRAP ${ }^{\circledR} / \mathrm{MS} 2$ combined with chemometrics methods," Amino Acids, vol. 47, no. 8, pp. 1589-1603, 2015.

[29] G. S. Zhou, Y. C. Yuan, Y. Yin et al., "Hydrophilic interaction chromatography combined with ultrasound-assisted ionic liquid dispersive liquid-liquid microextraction for determination of underivatized neurotransmitters in dementia patients'urine samples," Analytica Chimica Acta, vol. 1107, pp. 74-84, 2020.

[30] C. Q. Fu, P. Yu, M. Y. Wang, and F. Qiu, "Phytochemical analysis and geographic assessment of flavonoids, coumarins and sesquiterpenes in Artemisia annua L. based on HPLCDAD quantification and LC-ESI-QTOF-MS/MS confirmation," Food Chemistry, vol. 312, pp. 1260-1270, 2020.

[31] C. Hui, L. Zan, and T. Chao, "Fast discrimination of the geographical origins of notoginseng by near-infrared spectroscopy and chemometrics," Journal of Pharmaceutical and Biomedical Analysis, vol. 161, pp. 239-245, 2018.

[32] A. M. Ali, M. Burleigh, E. Daskalaki, T. Zhang, C. Easton, and D. Watson, "Metabolomic profiling of submaximal exercise at a standardised relative intensity in healthy adults," Metabolites, vol. 6, no. 1, p. 9, 2016. 